\title{
Under sulfur's spell
}

Thomas Rauchfuss marvels at the diversity of sulfur reactivity. Although it poisons most industrial catalysts, it adopts many forms in nature and takes on a variety of biological roles - including that of a biocatalyst.

W

hat is sulfur? This is a tricky

question, because many species with the formula $\mathrm{S}_{x}$ are known.

The most stable at room temperature is the crown-shaped ring $\mathrm{S}_{8}$, but elemental sulfur also contains small amounts of the brightyellow $\mathrm{S}_{7}$ and tiny amounts of other rings. On heating, sulfur readily converts to a metastable one-dimensional elastomer, which quickly degrades at room temperature back to the $\mathrm{S}_{8}$ form. The tendency of sulfur to form rings and chains (to catenate) is its most distinctive property. Only at high temperatures does sulfur form $\mathrm{S}_{2}$, the analogue of $\mathrm{O}_{2}$.

The catenating tendencies of sulfur are also evident in its anions - the polysulfides with formula $\mathrm{S}_{x}{ }^{2-}$. They arise by simply adding small amounts of reducing agents to elemental sulfur. These chains of sulfur atoms, which resemble alkanes in their conformation, can be extended or further shortened by redox reactions, a process that is exploited in the sodium-sulfur battery. The anionic chains exist in dynamic equilibrium with radicals such as $\mathrm{S}_{3}{ }^{--}$- the blue chromophore in lapis lazuli. The anions that terminate these chains can be capped with alkylating agents, protons and metal cations ${ }^{1}$. With metals, one obtains compounds with such improbable formulae as $\mathrm{PtS}_{15}{ }^{2-}$ and $\mathrm{Fe}_{2} \mathrm{~S}_{12}{ }^{2-}$.

Sulfur is readily oxidized, notably to dioxide and trioxide species. Depending on their environment, microorganisms subsist on the hydrolysed derivatives of these oxides through anaerobic respiration. Sulfur dioxide, an electrophilic, bent analogue of carbon dioxide, is produced on a massive, even weather-changing, scale by volcanic eruptions. Further oxidation gives the trioxide precursor to sulfuric acid, produced annually on the scale of about 140 megatons.

Sulfur is featured in two coded amino acids, methionine, which is fairly boring to the sulfur chemist, and cysteine, which has spectacularly diverse roles. By forming $\mathrm{S}-\mathrm{S}$ bonds, the conversion of cysteine to

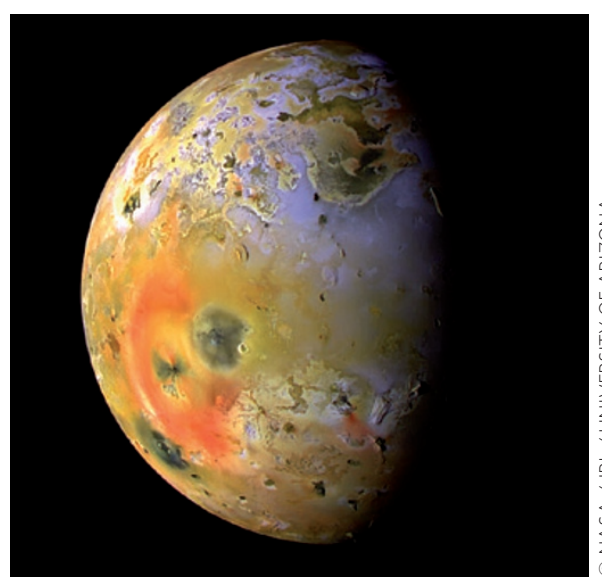

lo, a moon of Jupiter, has diverse colours owing to the presence of various sulfur species.

cystine rigidifies proteins in a manner similar to the crosslinking provided by the $\mathrm{S}-\mathrm{S}$ bonds in vulcanized rubber. Otherwise, the thiol group is a favoured site for posttranscriptional modifications. Sulfur is featured in many cofactors and vitamins, for example, thiamine, biotin and lipoic acid. Elucidation of their biosyntheses has been a trove of novel mechanisms.

As sulfur has occurred in proteins for millennia, fossil fuels often contain a few per cent by weight of organosulfur compounds, most problematically in the form of thiophenes. A continuing focus in petroleum refining is the removal of this sulfur, especially in view of the 5 ppm allowed limit of sulfur in many diesel fuels. This technology, called hydro-desulfurization, relies on modified molybdenum sulfide catalysts to yield a lowsulfur product and hydrogen sulfide waste. Oxidation of the waste produces vast amounts of sulfur, which beckons for new applications.

Thiolates exhibit particular affinity for metal ions; in enzyme active sites metals are commonly anchored via cysteinyl residues. The resilience of the metal-thiolate bond is exploited in the role of thiols in the formation of self-assembled monolayers. These materials are formed by simply contacting thiols with gold surfaces. Self-assembled monolayers are crucial in nanotechnology because they provide a responsive interface between mobile phases (gases and liquids) and an electrically conductive device.

The role of metal-sulfur bonds in biocatalysis is a popular area of research. One of the challenges is to elucidate the mechanism of methanogenesis - the source of the majority of Earth's natural gas and a contributor to climate change. The final stage of methanogenesis involves scission of a methyl-sulfur bond in coenzyme-M (first isolated from the sewers of Urbana, my home town). The allure of this area is heightened by the discovery that methanogenesis can run in reverse, through a nickel-catalysed reaction of methane ${ }^{2}$.

Transformations relevant to 'bioenergy' are effected by $\mathrm{Fe}-\mathrm{S}$ clusters, including the conversions of $\mathrm{CO}_{2}$ to $\mathrm{CO}$, of protons to $\mathrm{H}_{2}$, and of $\mathrm{N}_{2}$ to $\mathrm{NH}_{3}$ (ref. 3). These processes all involve the coordinated movement of protons and electrons - a mechanistic theme of immense significance. The active sites of the relevant enzymes consist of metal clusters where sulfide ligands help 'glue' together the metals. Because changes in electron count barely affect their structures, metal-sulfide clusters are able to quickly accept and relay electrons, as required for efficient catalysis. Apparently the presence of the soft sulfur ligands also enables the metal centres in these catalysts to bind and thereby activate weakly basic substrates such as $\mathrm{H}_{2}, \mathrm{CO}_{2}$ and $\mathrm{N}_{2}$ ligands normally reserved for organometallic compounds. Our ability to master these transformations could be key to our future. $\square$

THOMAS RAUCHFUSS is at the Department of Chemistry, University of Illinois, 600 South Mathews Avenue, Urbana, Illinois 61801, USA e-mail: rauchfuz@illinois.edu

\section{References}

1. Devillanova, F. A. (ed.) Handbook of Chalcogen Chemistry: New Perspectives in Sulfur, Selenium and Tellurium (Royal Society of Chemistry, 2006)

2. Thauer, R. K. Angew. Chem. Int. Ed. 49, 6712-6713 (2010).

3. Fontecilla-Camps, J. C., Amara, P., Cavazza, C., Nicolet, Y. \& Volbeda, A. Nature 460, 814-822 (2009).

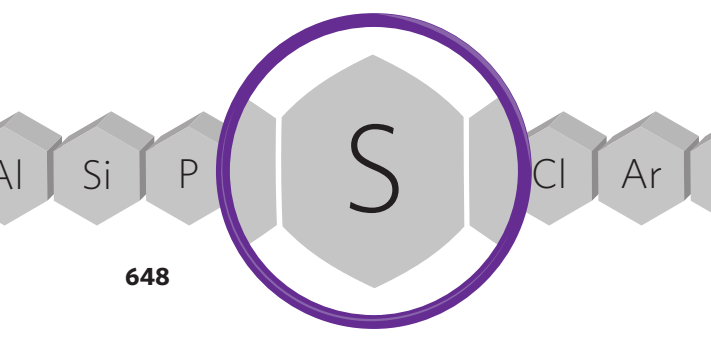

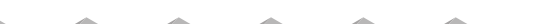

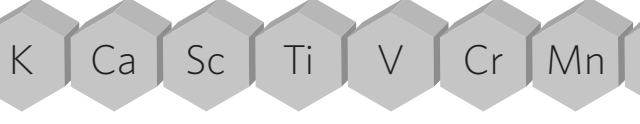

(6)
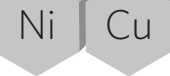